\title{
Recurrence of progressive multifocal leukoencephalopathy despite immune recovery in two HIV seropositive individuals
}

\author{
Kate M. Crossley ${ }^{1}$ (1) - Shruti Agnihotri ${ }^{2} \cdot$ Joga Chaganti $^{3} \cdot$ Michael L. Rodriguez $^{4}$. \\ Leon Patrick McNally ${ }^{5}$ Nagagopal Venna ${ }^{6} \cdot$ Sarah E. Turbett $^{7} \cdot$ Matthew Gutman $^{8}$. \\ Adrienne Morey ${ }^{9} \cdot$ Igor J. Koralnik $^{2}$ • Bruce J. Brew ${ }^{10,11}$
}

Received: 23 July 2015 /Revised: 3 December 2015 /Accepted: 16 December 2015 /Published online: 4 January 2016

(C) The Author(s) 2016. This article is published with open access at Springerlink.com

\begin{abstract}
We present two cases of recurrent progressive multifocal leukoencephalopathy (PML) in patients with long standing virally suppressed human immunodeficiency virus (HIV) and normal CD4+ T cell count who were taking stable regimens of highly active antiretroviral therapy (HAART). This has significant implications for other patients with a past history of PML, not just those with HIV but also those on medications such as natalizumab or fumarates.
\end{abstract}

Keywords Progressive multifocal leukoencephalopathy . Neuroimmunology $\cdot$ Neurovirology

\section{Introduction}

Progressive multifocal leukoencephalopathy (PML) usually affects immunocompromised people, such as those with advanced HIV or a haematological malignancy. A minority of

Kate M. Crossley

kate.crossley@health.nsw.gov.au

1 Department of Neurology, St Vincent's Hospital, Level 4 Xavier Building, Sydney 2010, Australia

2 Division of Neuro Immunology, Center for Virology and Vaccine Research, Beth Deaconess Medical Center, Boston, USA

3 Department of Radiology, St Vincent's Hospital, Sydney, Australia

4 Department of Neuropathology, St Vincent's Hospital, Sydney, Australia

5 Department of Molecular Medicine, St Vincent's Hospital, Sydney, Australia

6 Department of Neurology, Massachusetts General Hospital, Boston, USA initial PML diagnoses are in people who are minimally or mildly immunosuppressed (Berger et al. 1998; Gheuens et al. 2010). Management is to restore immune function where possible, and this can result in remission of the PML. The two cases we describe had initial remission of PML, with subsequent development of symptoms and investigation findings consistent with recurrent PML despite interval maintenance of minimal immunosuppression and HIV suppression. This has not previously been described.

\section{Case one}

A 49 year old man presented with chronic headache in 2013, on a background of HIV diagnosed in 1987.

In the early 2000s his CD4+ T cell count was at a lifetime nadir of 70 cells $/ \mu 1$, plasma HIV RNA $5 \cdot 35 \mathrm{log}$, and cerebrospinal fluid (CSF) 4.69 log. At that time he had cognitive

7 Department of Infectious Diseases, Massachusetts General Hospital, Boston, USA

8 Department of Neurosurgery, St Vincent's Hospital, Sydney, Australia

9 Department of Anatomical Pathology, St Vincent's Hospital, Sydney, Australia

10 Department of Neurology, St Vincent's Hospital, Sydney Australia, also Peter Duncan Neurosciences Unit Neurosciences Program, St Vincent's Centre for Applied Medical Research, Sydney, Australia

11 Department of Infectious Diseases and Immunology, St Vincent's Hospital, Sydney Australia, also Peter Duncan Neurosciences Unit Neurosciences Program, St Vincent's Centre for Applied Medical Research, Sydney, Australia 
impairment consistent with HIV associated neurocognitive disorder (HAND) with an MRI brain showing multifocal grey and white matter hyperintensities. CSF was normal including negative PCR for JC virus (JCV) DNA, but a right frontal lobe biopsy showed oligodendrocyte nuclei with viral inclusions, prominent reactive astrocytes including bizarre forms, and was strongly positive for JCV DNA by PCR. HIV associated encephalitis was also present (Anders et al. 1986).

He commenced HAART for the first time with efavirenz, lopinavir/ritonavir, lamivudine, and stavudine (the latter two later switched to raltegravir for tolerability), with consequent CD4+ T cell count rise to over 300 cells $/ \mu$ l. He was clinically stable but required institutional care due to poor cognition that did not improve with HAART.

By 2013 his CD4+ T cell count had been over 500 cells $/ \mu 1$ for 7 years, and HIV RNA had been undetectable for a decade. Annual MRI brain scans since 2008 (Figs. 1, 2 and 3) showed progressive white matter atrophy, and since 2010 asymptomatic fluctuating contrast enhancement without mass effect, in both cortical grey and subcortical white matter.

Examination when he presented with headache in 2013 revealed a new right inferior quadrantanopia, preexisting cognitive impairment, mild upper limb action tremor, and impaired tandem gait. CD4+ T cell count was 648 cells $/ \mu 1$. CSF was bland; cryptococcal antigen and HIV RNA were undetectable. However CSF JCV DNA was detected (below 30 copies $/ \mathrm{mL}$, the lower limit of quantification of the assay), using primers targeting the VP2 gene. The sequenced 131 base pair PCR product was a $93 \%$ match to JCV CPN1 strain using the Basic Local Alignment Search Tool. MRI brain

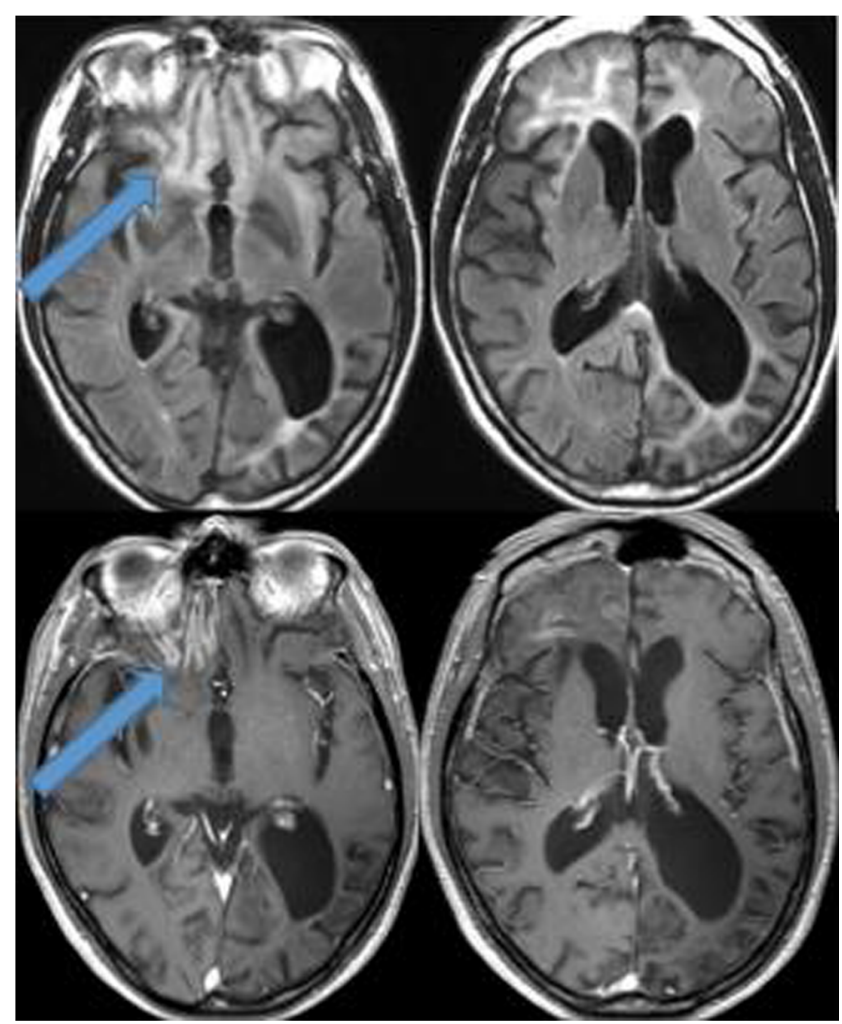

Fig. 2 Case one, 2011: FLAIR MRI sequences from 2011 again show hyperintensity in the right basifrontal (see arrows) and left parietooccipital regions, now with enhancement on corresponding post contrast $\mathrm{T} 1$ images (lower row) in the right basifrontal region only

showed increased subcortical signal hyperintensity and cortical enhancement in the left posterior temporal lobe extending
Fig. 1 Case one, 2008: Fluid attenuated inversion recovery (FLAIR) MRI sequences showing multifocal hyperintensity in the subcortical U-fibres of the right basifrontal, right temporal, and left parieto-occipital regions (see arrows). The corresponding T1 post contrast images (lower row), demonstrate an absence of enhancement

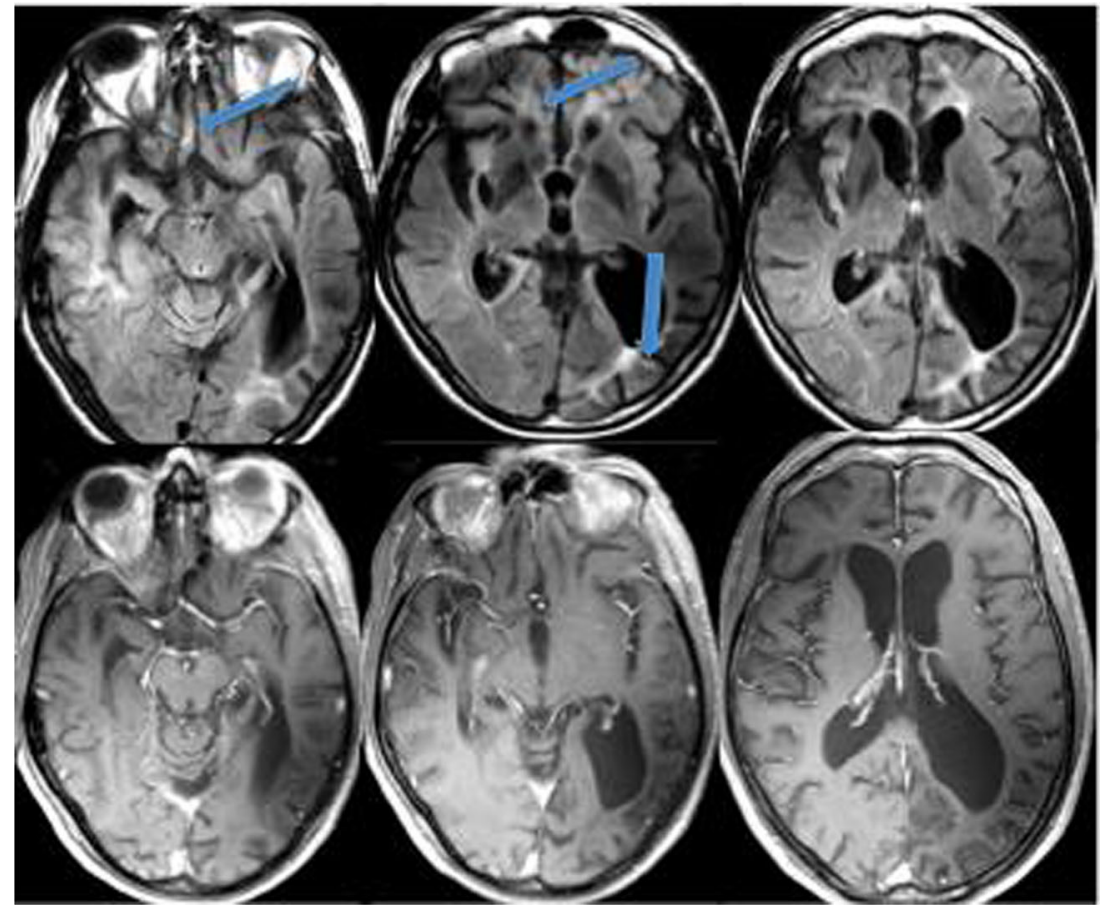




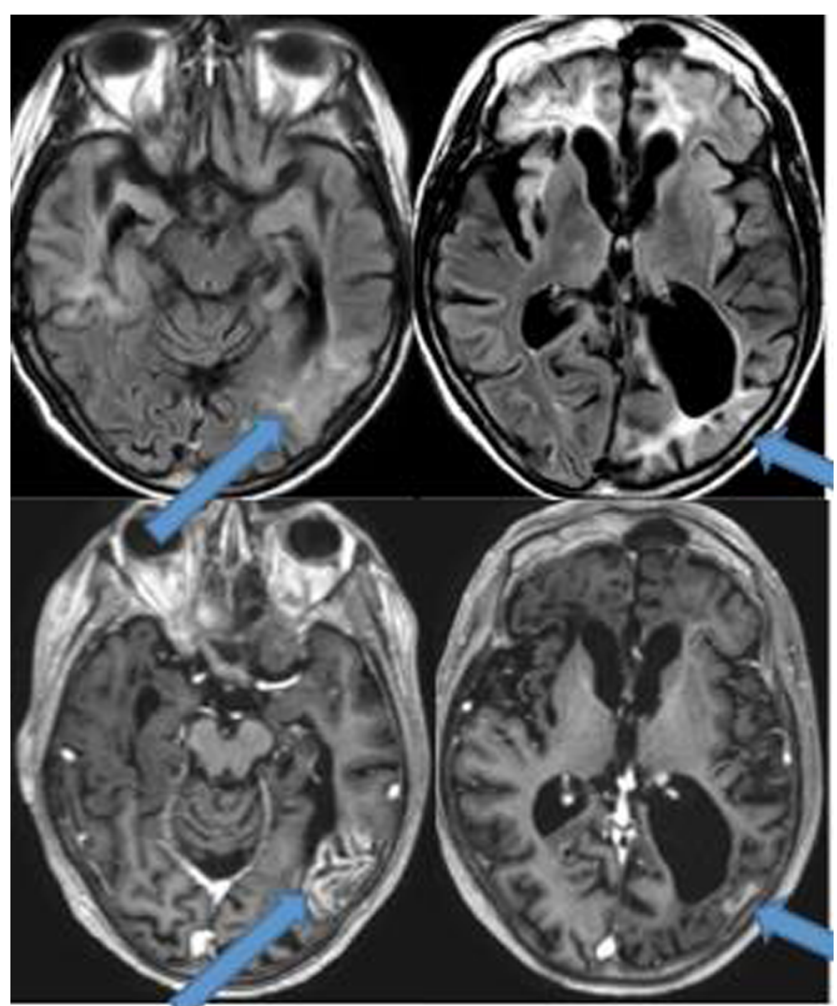

Fig. 3 Case one, 2013: FLAIR hyperintensity has increased in the left parieto-occipital region (see arrows, top images), with new gyriform enhancement (see arrows, bottom images) on corresponding precontrast FLAIR sequences. The right basifrontal contrast enhancement has shown a complete resolution

into the parieto-occipital region (Fig. 3). He was diagnosed with possible recurrent PML.

His headache spontaneously improved only to recur several months later. An MRI showed new enhancing lesions in the left temporoparietal grey and subcortical white matter. Serial MR spectroscopic studies showed increasing choline and myoinositol in these regions with markedly reduced Nacetylaspartate implying ongoing gliosis of the subcortical U-fibres. CSF again showed low JC viral load. A stereotactic-guided biopsy of the left temporoparietal lesion was performed within a month of presentation, and repeated six months later because of the unusual findings, with essentially the same result. The patient has shown no clinical progression on history or examination since the biopsies. MRI has not been repeated.

\section{Pathological findings}

The leptomeninges were fibrotic with increased vascularity and patchy chronic inflammation, predominantly $\mathrm{T}$ cells. The cortex displayed prominent reactive gliosis, microglial activation with multifocal microvascular proliferation, mild neuronal loss, demyelination with partial axon preservation, sparse T cells (predominantly CD8+), and some haemosiderin deposition. There were scattered dysmorphic neurons, some with enlarged nuclei, marginated chromatin, and peripherally displaced Nissl substance, and several bizarre astrocytes with enlarged hyperchromatic pleomorphic nuclei.

There were frequent polyomavirus immunoreactive nuclei mostly in astrocytes and occasional neurons (SV40 large T antigen $\mathrm{Ab}$ cross-reacting with JCV). Most of the positive nuclei were not within the areas of demyelination or microvascular proliferation. Classic oligodendroglial inclusions and microglial nodules, noted in the first biopsy, were not identified. Immunostains for HIV p24, CMV, Toxoplasma, and HHV8 were negative.

\section{Case two}

A 54-year-old female reported new gait difficulties and unsteadiness in 2012, with associated recent decline in memory and language.

In 2006 HIV was diagnosed after presenting with memory impairment. At the time her nadir $\mathrm{CD} 4+\mathrm{T}$ cell count was 186 cells $/ \mu 1$ with an HIV RNA viral load of $5 \cdot 28 \log$. An MRI showed left frontotemporal white matter lesions (Fig. 4). CSF was qualitatively positive for JCV DNA by PCR. No quantitative test was performed. She improved significantly on a stable regimen of tenofovir, emtricitabine, and efavirenz. Her HIV RNA was below detection on all subsequent testing.

MRI in 2012 showed significant atrophy (predominantly left frontoparietal) and new non-enhancing T2 hyperintensities in the middle cerebellar peduncles, splenium and periventricular region (Fig. 5), mainly involving white matter, but also grey-white matter junction.

CSF was positive for JCV DNA at 2786 copies $/ \mathrm{ml}$ and negative for HIV RNA. She had a detectable immune response to JCV mediated by both CD4+ and CD8+ T cells. Although her CD4+ T cell count was 676 cells $/ \mu$ and HIV RNA undetectable, her medications were changed to abacavir, emtricitabine, etravirine, raltegravir, and maraviroc to optimise brain penetration. The right frontal region was biopsied. Mirtazapine was trialled to no avail, and she deteriorated and died seven months after the biopsy.

\section{Pathological findings}

The biopsy showed marked reactive white matter gliosis, and acute demyelination. There were scattered large, atypical nuclei, some suggestive of viral inclusions. Immunostaining using SV40 Ab highlighted scattered positive cells, mostly oligodendrocytes in the white matter edge of the demyelinating lesions, and the grey-white junction. 


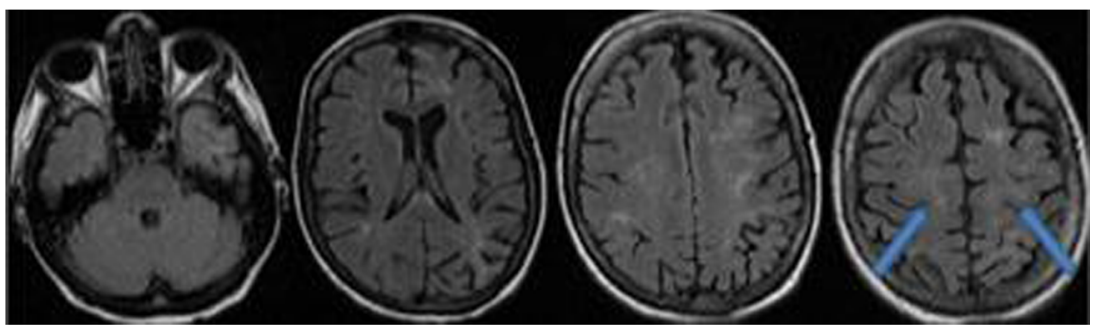

Fig. 4 Case two, 2006: FLAIR MRI sequences demonstrating multiple poorly defined bright foci in the central and periventricular white matter, with subcortical U-fibre involvement (see arrows) in the frontoparietal region

HIV encephalitis was present with marked microglial activation (highlighted with CD45 immunostaining) occasional microglial nodules and reactive gliosis with mild neuronal loss (highlighted with NeuN). Additionally there was mild chronic inflammation, with perivascular and scattered parenchymal $\mathrm{CD} 4+, \mathrm{CD} 8+$, and CD3+ T cells which could not be accurately quantified because of the limited tissue available for study.

\section{Discussion}

These cases demonstrate three novel important points. First, PML may recur years after the initial illness, despite normal or near-normal immune function. Second, recurrence of clinical PML may be presaged by MRI brain changes. Third, the MRI changes may fluctuate over time.

We believe JCV caused the clinical, radiological, and neuropathological features described. In both cases, clinical and MRI findings were compatible with PML (Brew et al. 2010; Gheuens et al. 2013), although in the first case, the fluctuating cortical gyriform enhancement is unusual. The pathology was also consistent with PML except the microvascular proliferation. Hyperperfusion within and at the edge of PML lesions has previously been demonstrated, however it was unclear whether this was due to microvascular proliferation or vasodilation (Khoury et al. 2013).
SV40 staining was seen in cortical neurons in the first case. This has been described in up to half of patients with classic PML, within areas of cortical demyelination or in isolation (Wuthrich and Koralnik 2012). Our patient's biopsy showed cortical demyelination with infection of astrocytes and neurons and some inflammation. This is consistent with cortical involvement by PML, which may explain the cortical gyriform enhancement seen on MRI.

Most importantly, JCV DNA was detected in the CSF and brain of both patients by several techniques. No alternative cause was found. Autopsies of HIV infected patients have previously shown "burnt out" cerebral lesions from long term PML without evidence of JCV activity (Gray et al. 2003). There is no clear reason why these two patients in particular had PML recurrence; hypotheses include an undetermined host genetic predisposition, HIV-accelerated age related immune senescence, or viral factors such as development of a mutation in a JCV quasi-species.

JCV DNA has previously been found in low copy number in CSF up to one half of natalizumab associated PML patients months (and in one case three years) after PML stabilization off natalizumab (Ryschkewitsch et al. 2010). However those patients had no definite evidence of clinically active disease in contradistinction to our two patients.

These cases therefore have significant implications for the prognosis and management of patients with JCV CNS infection. Although PML often improves with HAART, our

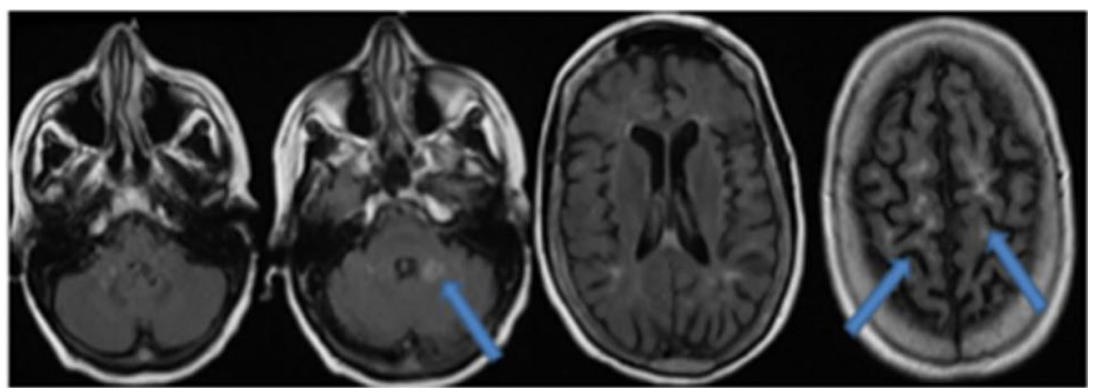

Fig. 5 Case two, 2012: FLAIR MRI sequences showing new lesions in the posterior fossa (see arrow) and an increase in the number of lesions in the high frontoparietal white matter bilaterally (see arrows). There was also increased cerebral atrophy. There was no mass effect, perilesional oedema, or enhancement after contrast administration (not shown) 
findings suggest this population, and others with PML, may be at risk of PML recurrence. Serial monitoring clinically and by MRI may allow early identification. There is no proven PML therapy besides immune reconstitution (Tan et al. 2009; Koralnik 2006), which is of particular concern given that our patients were not immunosuppressed anymore. However, should PML recurrence be identified in other groups such as natalizumab or fumarates-treated patients, current immunomodulating and immunosuppressive therapies may need to be reconsidered.

\section{Compliance with ethical standard}

Conflict of Interests Igor J. Koralnik was supported in part by NIH grants R01 047029 and R01 074995. The other authors all declare that they have no conflict of interest.

Ethics Ethics approval was not required for this study.

Contributorship statement $\mathrm{KC}, \mathrm{SA}, \mathrm{NV}, \mathrm{ST}, \mathrm{IK}$ and BB identified and managed the two cases. $\mathrm{KC}, \mathrm{SA}, \mathrm{IK}$ and $\mathrm{BB}$ wrote the first draft. $\mathrm{JC}, \mathrm{MR}, \mathrm{LM}, \mathrm{MG}$ and AM were responsible for radiological (JC), surgical (MG) and pathological (LM, MR, AM) management and interpretation, and contributed these sections to the manuscript. All authors contributed substantially to reviewing, revising, and finalising the manuscript.

Funding This research received no specific grant from any funding agency in the public, commercial or not-for-profit sectors.

Declaration Dr Crossley affirms that the manuscript is an honest, accurate, and transparent account of the study being reported, and that no important aspects of the study have been omitted.

Licence and copyright The corresponding author has the right to grant on behalf of all authors and does grant on behalf of all authors, a worldwide licence to the publishers and its licensees in perpetuity, in all forms, formats and media (whether known now or created in the future), to (i) publish, reproduce, distribute, display and store the contribution,( ii) translate the contribution into other languages, create adaptations, reprints, include within collections and create summaries, extracts and/or, abstracts of the contribution, (iii) create any other derivative work(s) based on the contribution, (iv) to exploit all subsidiary rights in the contribution, (v) the inclusion of electronic links from the contribution to third party material where-ever it may be located; and, (vi) licence any third party to do any or all of the above. Copyright is retained by the authors of this article.
Open Access This article is distributed under the terms of the Creative Commons Attribution 4.0 International License (http:// creativecommons.org/licenses/by/4.0/), which permits unrestricted use, distribution, and reproduction in any medium, provided you give appropriate credit to the original author(s) and the source, provide a link to the Creative Commons license, and indicate if changes were made.

\section{References}

Anders KH, Guerra WF, Tomiyasu U, Verity MA, Vinters HV (1986) The neuropathology of AIDS. UCLA experience and review. Am J Path 124:537-558

Berger JR, Pall L, Lanska D, Whiteman M (1998) Progressive multifocal leukoencephalopathy in patients with HIV infection. J Neurovirol 4(1):59-68

Brew BJ, Davies NWS, Cinque P, Clifford DB, Nath A (2010) Progressive multifocal leukoencephalopathy and other forms of JC virus disease. Nat Rev Neurosci 6:667-679

Gheuens S, Pierone G, Peeters P, Koralnik I (2010) Progressive multifocal leukoencephalopathy in individuals with minimal or occult immunosuppression. J Neurol Neurosurg Psychiatry 81(3):247-254

Gheuens S, Wuthrich C, Koralnik I (2013) Progressive multifocal leukoencephalopathy: why gray and white matter. Annu rev pathol mech dis 8:189-215

Gray F, Chretien F, Vallat-Decouvelaere AV, Scaravilli F (2003) The changing pattern of HIV neuropathology in the HAART era. J Neuropathol Exp Neurol 62(5):429-440

Khoury M, Gheuens S, Ngo L, Wang X, Alsop D, Koralnik I (2013) Hyperperfusion in progressive multifocal leukoencephalopathy is associated with disease progression and absence of immune reconstitution inflammatory syndrome. Brain 136:3441-3450

Koralnik IJ (2006) Progressive multifocal leukoencephalopathy revisited: has the disease outgrown its name? Ann Neurol 60:162-173

Ryschkewitsch CF, Jensen PN, Monaco MC, Major EO (2010) JC Virus persistence following PML in MS patients treated with natalizumab. Ann Neurol 68(3):384-391

Tan K, Roda R, Ostrow L, McArthur J, Nath A (2009) PML-IRIS in patients with HIV infection: clinical manifestations and treatment with steroids. Neurology 72:1458-1464

Wuthrich C, Koralnik I (2012) Frequent infection of cortical neurons by JC virus in patients with progressive multifocal leukoencephalopathy. J Neuropathol Exp Neurol 71(1):54-65 